\title{
A Scatter Search Method for the Multidimensional Knapsack Problem with Generalized Upper Bound Constraints
}

\author{
Vincent C. Li ${ }^{1}$, Yun-Chia Liang ${ }^{2, *}$, Yu-Yen Sun ${ }^{3}$, You-Shan Chen ${ }^{4}$
}

\begin{abstract}
In this paper, a scatter search (SS) heuristic is proposed to solve the multidimensional knapsack problem with generalized upper bound constraints (GUBMKP). The method is organized according to the general structure of SS. We discuss the design and implementation for each of the components of SS. A greedy randomized adaptive search procedure is applied in order to diversify the initial solutions. In order to select diversified solutions to enter the reference set, we propose an algorithm based on the structure of generalized upper bound constraints. Several approaches of combining solutions are proposed to solve the problem. The computational results show the heuristic is competitive compared to the former leading method for the GUBMKP.
\end{abstract}

Keywords: multidimensional knapsack problem, generalized upper bound, scatter search

\section{Introduction}

In combinatorial optimization, the multidimensional knapsack problem (MKP) has been studied extensively. The formulation of MKP can be described as follows.

\section{Formulation 1 (MKP).}

$$
\begin{array}{ll}
\max & \sum_{j=1}^{n} c_{j} x_{j} \\
\text { s.t. } & \sum_{j=1}^{n} a_{i j} x_{j} \leq b_{i}, i=1, \cdots, m . \\
& x_{j} \in\{0,1\}, \quad j=1, \cdots, n .
\end{array}
$$

\footnotetext{
*Corresponding author. Fax: 886-3-4638907, TEL: 886-3-4638800\#2521

Email address: ycliang@saturn.yzu.edu.tw (Yun-Chia Liang)

${ }^{1}$ Department of Business Administration, National Chiayi University, Chiayi, Taiwan 60054

${ }^{2}$ Department of Industrial Engineering and Management, Yuan Ze University, Taoyuan, Taiwan 32003

${ }^{3}$ Department of Computer Science and Information Engineering, National Dong Hwa University, Hualien, Taiwan 97401

${ }^{4}$ Graduate Institute of Logistics Management, National Dong Hwa University, Hualien, Taiwan 97401
} 
In this formulation, coefficients $c_{j}, a_{i j}$, and $b_{i}$ are all assumed to be nonnegative. In addition, $\max _{j=1}^{n}\left\{a_{i j}\right\} \leq b_{i}$ for $i=1,2, \cdots, m$. Many applications of the MKP are to allocate resources, such as cutting stock [8], project selection [33], loading [36], and scheduling of satellite photographs [37]. Meier et al. [31] also included the MKP as a subproblem for project evaluation. In some applications, all the $n$ decision variables $x_{j}$ in the MKP can be partitioned into $K$ disjoint groups, and at most one decision variable can be chosen in each group. In this case, each group of variables associated with a subset $S_{k}$ forms a generalized upper bound constraint with an upper bound of 1 as shown in Eq. 4.

$$
\begin{gathered}
\sum_{j \in S_{k}} x_{j} \leq 1, k=1, \cdots, K, \\
\text { where }\left|\bigcup_{k=1}^{K} S_{k}\right|=n,
\end{gathered}
$$

$$
\begin{aligned}
& \text { and } S_{k_{1}} \bigcap S_{k_{2}}=\emptyset, \\
& \qquad 1 \leq k_{1}, k_{2} \leq K, k_{1} \neq k_{2} .
\end{aligned}
$$

The formulation of the multidimensional knapsack problem with generalized upper bound constraints (referred to the GUBMKP) consists of Eqs. 1 to 6 . These groups are mutually exclusive and totally exhaustive as shown in Eqs. 5 and 6. Utilizing this formulation, Li et al. [25] formulated a strike force asset allocation problem for the US Navy, and Wilhelm and Gokce [39] developed a surveillance system for port and waterway security.

It is worth mentioning here that there is another variant of the MKP very similar to the GUBMKP, where the restriction of picking at most one item from each group is tightened to be exactly one item. This class of the problem is referred to the multidimensional multiple-choice knapsack problem (MMKP). There are numerous applications of the MMKP such as real-time multimedia domain problems [32].

The well-known MKP is NPHard [29]. As the MKP can be reduced to the GUBMKP by forming a GUB constraint for each variable, the GUBMKP is also NP-Hard. Metaheuristics have been proved effective in tackling a lot of NP-Hard problems. The focus of this paper is to develop a scatter search (SS) method for the GUBMKP. Scatter search is an evolutionary metaheuristic that originates from the idea of creating the surrogate constraint due to Glover [9]. Instead of combining constraints, SS combines elite solutions from a reference set systematically. The principle of building the reference set is to make sure the elite solutions either have high-quality or have high-diversity. As Glover et al. [15] pointed out, useful information can be found from a diverse collection of elite solutions. To take advantage of such information, it is important to make combinations of the elite solutions such that the produced solution is not limited to the region spanned by the elite solutions. Glover et al. [15] commented that having diversity in the combined solution does not guarantee the quality, so improvement effort is needed after the combination process.

In order to provide a convenient basis for implementing SS, Glover [11] presented a template that consists of five main components. Our SS heuris- 
tic is developed based on these five elements: (1) a diversification generation method to create a collection of diverse trial solutions; (2) an improvement method to enhance the trial solutions; (3) a reference set update method to construct and update the reference set; (4) a subset generation method to form subsets of solutions according to the reference set; (5) a solution combination method to produce one or more new solutions from the subsets generated in the previous element.

The remainder of this paper is organized as follows. Section 2 reviews the literature for the GUBMKP. Section 3 discusses the SS methodology and outlines our heuristic implementation for each of the five components. Computational results are shown in Section 4. Conclusions and future directions are discussed in Section 5.

\section{Literature review}

The GUBMKP is a close variant of the MKP, that has been tackled by various techniques such as exact methods, approximation algorithms, dynamic programming, heuristics, and metaheuristics. The reader can refer to Fréville [6] for a review of the existing approaches for the MKP, and Wilbaut et al. [38] and Crévits et al. [3] for variants of knapsack problems. In particular, the multiobjective MKP has gained more attention recently. For examples, Lust and Teghem [28] provided a comprehensive review, and Florios et al. [5] proposed evolutionary algorithms to solve this class of problem.

The MMKP has been tackled by using heuristics (e.g., [32]). Metaheuristics, such as tabu search, have been utilized to solve the MMKP as well (e.g., [20]). For a more detailed review of the MMKP, see Hiremath and Hill [21].

As to the GUBMKP, a critical event tabu search method (CETS), originated from Glover and Kochenberger [14] for solving the MKP, was adapted in Li and Curry [26]. The CETS method consists of a constructive phase (adding variables) and a destructive phase (dropping variables), and the method oscillates around the feasibility boundary with systemcontrolled depth. Critical solutions obtained while crossing the boundary are kept in tabu memory. Critical solutions are important because they are near the feasibility boundary. To explore the neighborhood around the critical solutions, Li [24] proposed a tight oscillation scheme which considers the changes of both the objective function and feasibility in its choice rules. This oscillation swings back and forth around the feasibility boundary very closely till a duplicated solution has been found.

By adopting the adaptive memory projection framework in Glover [13], $\mathrm{Li}$ et al. [27] implemented a method that iteratively combines critical event tabu search with an MIP solver for the GUBMKP. We develop a scatter search heuristic for the GUBMKP in this study. SS has been applied to a fairly large number of combinatorial optimization problems. Particularly, SS has been shown to be effective in dealing with a variety of knapsack variants, such as $[1,7,18,19,22]$. Gomes da Silva et al. [18] proposed an SS method for finding a good approximation of the non-dominated frontier for bi-criteria binary knapsack instances. In the diversification component of their SS method, the mul- 
tiple knapsack constraints were converted into a surrogate constraint, and the surrogate multipliers were determined by a subgradient-like method. Hvattum and Lokketangen [22] used SS to solve the multidemand multidimensional knapsack problem. In their paper, a standard diversification generation method was implemented and then a flip-neighborhood that consists of all solutions of hamming distance one from the current solution was used for improving the generated solution. Hanafi and Wilbaut [19] applied scatter search to the MKP; in particular, they designed a relaxation-based diversification generator to produce an initial population with elite solutions. Beausoleil et al. [1] utilized multi-start search (see Glover [12]) and path relinking (the generalization of scatter search [15]) to lead the search to the most balanced zone of Pareto optimum for a multiobjective knapsack problem. Gholipour-Kanani et al. [7] tackled a multicriteria group scheduling problem for a cellular manufacturing system by scatter search. Our paper differs from the previous scatter search knapsack applications $[17,23]$ by (1) utilizing GRASP to populate reference-set solutions, (2) introducing different diversity measurement to capture the GUB structure, and (3) incorporating the benefit-cost ratio (referred to the $\mathrm{B} / \mathrm{C}$ ratio hereafter) when making crossover combination.

\section{Methodology}

Following the template in Glover [11], our scatter search for the GUBMKP consists of methods described in each of the following five subsections.

\subsection{A diversification generation method}

The goal of the diversification method is to generate a set of solutions which strikes a balance between solution quality and diversity for initializing and rebuilding the reference set used. The greedy randomized adaptive search procedure (GRASP) was used to generate a population of initial solutions. As Laguna and Martí [23] pointed out, the GRASP is usually implemented as a multi-start procedure which consists of a semi-greedy construction phase and a local search phase. A comprehensive review of GRASP can be found in Resende and Ribeiro [35]. Adapted from a generic pseudo code of GRASP by Feo and Resende [4], the construction phase of the proposed SS method is described following the description of the greedy function for GRASP.

\subsubsection{Greedy function for GRASP}

The greedy function $g$ is determined by the $\mathrm{B} / \mathrm{C}$ ratio of the corresponding variable. To calculate this ratio, a surrogate constraint needs to be generated first. In the ratio, the objective function coefficient represents the benefit whereas the surrogate constraint coefficient represents the cost. The surrogate constraint shown in Eq. 7 is obtained from a linear combination of all the knapsack constraints, where $s_{j}=\sum_{i=1}^{m} \mu_{i} a_{i j}$, $s_{0}=\sum_{i=1}^{m} \mu_{i} b_{i}$, and $\mu_{i}$ is the multiplier associated with the $i^{\text {th }}$ knapsack constraint.

$$
\sum_{j=1}^{n} s_{j} x_{j} \leq s_{0} .
$$

The GUB constraints are excluded from consideration when forming the surrogate constraint to preserve their 
simple structure. With the surrogate constraint, $\gamma_{j}$, the $\mathrm{B} / \mathrm{C}$ ratio for $x_{j}$ equals to $c_{j} / s_{j}$. The multiplier for each of the surrogate constraints is determined by Eq. 8 as suggested by Glover [10]:

$$
\begin{aligned}
\mu_{i}=\frac{\sum_{j \in \Phi} a_{i j}-b_{i}}{b_{i}}, \\
\quad i \in\{1, \cdots, m\},
\end{aligned}
$$

where $\Phi$ refers to the set of variables that are eligible to be added (a variable is in this set if it equals 0 and the corresponding GUB constraint is not tight). A GUB constraint is said to be tight or active if the equality in Eq. 4 holds. It is impossible to add any variable to be 1 if this GUB constraint is active as there is no slack any more. The multipliers generated this way are changed dynamically, so is the surrogate constraint.

In the constructive phase of each iteration, a value-based RCL is built from $r$ eligible elements (variable candidates) where $r$ is a variable. Some more notations are introduced as follows.

$\alpha$ : the threshold parameter with a value between 0 and 1 for the RCL,

$v$ : the variable index of the candidate element, where $v \in \Phi$,

$\bar{g}$ : the maximum greedy function value of all candidate elements, i.e., $\bar{g}=\max \{g(v) \mid v \in \Phi\}$,

$g$ : the minimum greedy function value of all candidate elements, i.e., $g=\min \{g(v) \mid v \in \Phi\}$.

The restricted candidate list can be stated as follows.

$$
\begin{aligned}
& \mathrm{RCL}= \\
& \{v \in \Phi \mid g(v) \geq \underline{g}+\alpha(\bar{g}-\underline{g})\} .
\end{aligned}
$$

The GRASP becomes a greedy heuristic if $\alpha$ equals 1 , whereas it becomes a completely random heuristic if $\alpha$ equals 0 . Prais and Ribeiro [34] commented that a fixed value of $\alpha$ may impede the process of searching better solutions. They proposed a reactive GRASP that self adjusts the value of $\alpha$ in the construction process. In our study, four fixed $\alpha$ values were tested. In addition, a random choice among the aforementioned values was also implemented for comparison.

\subsubsection{GRASP Procedure}

The GRASP procedure implemented in this study can be described as follows.

Step 0.1: Initialize $S$, where $S$ denotes the set of variables that equal to 1 . If the initial solution is not feasible yet, recover the feasibility prior to Step 1.

Step 0.2: Initialize the value of $\alpha$ for the greedy function.

Step 1: Based on $S$, determine the restricted candidate list (RCL), where the RCL consists of variables feasible to be added. Thus the resulting solution after the proposed addition satisfies all the knapsack and GUB constraints. Use the greedy function $g$ to evaluate the $\mathrm{B} / \mathrm{C}$ ratio of each of the variables in the RCL.

Step 2: While the RCL is not empty,

1. randomly select a variable $x_{j}$ from the RCL,

2. update $S=S \cup\left\{x_{j}\right\}$, 
3. change $\alpha$ if it is required. Update the greedy function $g$ if $\alpha$ has been changed,

4. update the RCL. The RCL is shrunk by removing two sets of variables: (1) variables in the same GUB set of $x_{j}$, and (2) variable $x_{k}$ if $a_{i k}>b_{i^{\prime}}$ where $a_{i k}$ is the coefficient of the $k^{\text {th }}$ variable in the $i^{\text {th }}$ knapsack constraint, and $b_{i^{\prime}}$ is the updated right-hand-side (RHS) value of the $i^{\text {th }}$ knapsack constraint.

Step 3: If the number of distinct solutions has reached the specified solution pool size, terminate this part. Otherwise, go to step 0.1 to construct another solution.

For simplicity, no variables are assigned to be 1 in the initial solution. Thus $S$ is an empty set initially, and this strategy is named as $\mathrm{G}_{0} . \mathrm{G}_{0}$ is similar to what Chardaire et al. [2] proposed in the MKP context. The difference lies in the limitation brought by the GUB constraints.

Given an RCL, a variable is chosen randomly in order to move closer to the feasibility boundary. The RCL is updated after a member is randomly selected from the list. Notice if adding a variable leads to violation of any GUB constraint or knapsack constraint, then this variable is excluded from the current candidate list. The candidate list becomes empty if there is no more feasible move in the neighborhood, and the current solution is treated as a "constructed" solution (i.e., $\Phi=\emptyset$ at this point) in the terminology of GRASP.

\subsection{An improvement method}

Once a constructed solution is obtained from the diversification generation method, it will go through the improvement method. The solutions obtained from either of the diversification or combination are already ensured feasible. The feasibility will be maintained throughout the improvement method. In order to improve the solution quality, swap moves that first select a variable to drop and then another variable to add are considered. Swapping is continued until no more improvement can be made in the neighborhood of the current solution. In evaluating which variable to drop, variables are sorted by the $\mathrm{B} / \mathrm{C}$ ratio in the non-decreasing order, and the sorting order is reversed when evaluating which variable to add. A pseudocode for the improvement method is shown in Algorithm 1.

\subsection{A reference set update method}

The reference set $R$ is divided into two subsets including $R_{1}$ and $R_{2}$. Subset $R_{1}$ contains $r_{1}$ solutions with the highest objective function values. Solutions in $R_{1}$ are sorted into a nonincreasing sequence based on their objective function values, and they are denoted as $R_{1}(1), R_{1}(2), \cdots, R_{1}\left(r_{1}\right)$.

Algorithm 2 is used to evaluate the diversity between two solutions. The diversity between two solutions $A$ and $B$ are calculated by summing up the "differences" of all the GUB sets. Denote $A_{k}$ and $B_{k}$ as the active-variable indexes in the $k^{\text {th }}$ GUB set of solutions $A$ and $B$, respectively. The index is set to -1 if the corresponding GUB constraint is not tight.

The relationship between $A_{k}$ and $B_{k}$ can be described by the following 
three cases. Case 1 applies when $A_{k}$ equals $B_{k}$. There is no difference in the $k^{\text {th }}$ GUB set. Case 2 occurs when both $A_{k}$ and $B_{k}$ are positive but not equal. These two solutions are similar to each other since this particular GUB constraint is tight in both of the solutions. However, they can still be considered different "to some degree" since the active variables in this GUB set are not exactly the same. Case 3 appears when only one of $A_{k}$ and $B_{k}$ is positive. The difference between these two solutions will be added by 1 .

Let $\Omega_{k}$ represent the $k^{\text {th }}$ GUB set. Define $\delta$ to be a multiplier between 0 and 1 that weights the difference in a particular GUB set. When $\delta$ is set to 0 , the difference in case 2 will simply be ignored. The diversity between solutions $A$ and $B$ (denoted as $\Delta(A, B)$ ) when $\delta$ is set to 0 can be represented by Eq. 10, and is computed by calling Procedure Diversity(A, B, 0):

$$
\begin{aligned}
& \Delta(A, B)= \\
& \Sigma_{\forall \Omega_{k}}\left|\mathbf{1}_{\{-1\}}\left(A_{k}\right)-\mathbf{1}_{\{-1\}}\left(B_{k}\right)\right|,
\end{aligned}
$$

where $\mathbf{1}_{S}(\cdot)$ is an indicator function of set $S$, i.e.,

$$
\mathbf{1}_{S}(w)= \begin{cases}1 & \text { if } w \in S \\ 0 & \text { otherwise }\end{cases}
$$

On the contrary, when $\delta$ is set to 1 , this difference in case 2 will be counted completely, and the diversity will be added by 2 since there are two different active variables. The diversity between solutions $A$ and $B$ when $\delta$ is set to 1 can be stated as Eq. 12:

$$
\begin{array}{r}
\Delta(A, B)=\Sigma_{j}\left|\left(x_{j}\right)_{A}-\left(x_{j}\right)_{B}\right|, \\
j=1, \cdots, n,
\end{array}
$$

where $\left(x_{j}\right)_{A}$ and $\left(x_{j}\right)_{B}$ refer to the value of $x_{j}$ in solutions $A$ and $B$, respectively. Eq. 12 is computed by calling Procedure Diversity (A, B, 1). $\delta$ can also be set to a fractional value between 0 and 1 in order to get a mixed strategy for evaluating the diversity. Subset $R_{2}$ contains $r_{2}$ solutions with the highest diversity scores. Solutions in $R_{2}$ are sorted into a non-increasing sequence based on their diversity values.

Once a new solution $x$ has been obtained after the solution combination method and the improvement method, then it will be compared with the lowest-ranked solution $y$ in subset $R_{1}$. If $x$ is better than $y$ in terms of the objective function value, then solution $x$ is admitted to subset $R_{1}$ while solution $y$ will be removed from this subset. As solution $y$ leaves the subset $R_{1}$, it will be compared with the lowestranked solution $z$ in subset $R_{2}$ in terms of the diversity value to see whether it can be admitted to subset $R_{2}$.

\subsection{A subset generation method}

In scatter search, solutions in the reference set can be grouped into various subsets. For each subset, various types of combination can be used to generate trial solutions. Glover et al. [16] suggested four types of subsets to be generated. This study utilizes the first type of subset that consists of all pairs of elements. With two reference subsets $R_{1}$ and $R_{2}$, there are totally $C_{2}^{\left|R_{1}\right|+\left|R_{2}\right|}$ pairs of solutions, where " $|S|$ " denotes the cardinality of a subset $S$. Notice if a pair of solutions has been combined before, it is not necessary to make the combination again. Therefore, a hashing structure that records some of the attributes of 
the combined pairs is used to prevent from duplicating the combination effort.

\subsection{A solution combination method}

In this study, four approaches of combining sets of solutions were developed. For simplicity, we choose to illustrate how to combine two solutions. These approaches can be easily generalized to combine three or more solutions. Let the $j^{\text {th }}$ variable of base solutions $A$ and $B$ as $\left(x_{j}\right)_{A}$ and $\left(x_{j}\right)_{B}$, respectively. The resulting solution is denoted as solution $D$. The combination of two solutions is done in the GUB sets one by one. There are three scenarios to be considered.

1. The GUB-set status equals -1 (inactive) in both solutions.

2. The GUB-set status is positive (active) in both solutions.

3. One of the GUB-set status is positive (active) while the other equals -1 (inactive).

The four combination approaches are described as follows.

1. (Combination by intersection, $\left.\mathrm{C}_{\mathrm{I}}\right)$ The first approach combines two base solutions by finding their intersection of 1-set elements. That is, $\left(x_{j}\right)_{C}$ equals 1 if and only if both $\left(x_{j}\right)_{A}$ and $\left(x_{j}\right)_{B}$ equal 1 . The relationship is stated in Eq. 13.

$$
\left(x_{j}\right)_{D}=\left(x_{j}\right)_{A}\left(x_{j}\right)_{B} .
$$

2. (Combination by union, $\mathrm{C}_{\mathrm{U}}$ ) The second approach combines two base solutions by finding their union of 1-set elements. However, if a GUB set is active in both solutions, the solution obtained from union will not be GUB-feasible unless the active variable is the same in both solutions. In this situation, we let the variable with bigger static $\mathrm{B} / \mathrm{C}$ ratio $\gamma$ have a $2 / 3$ chance to be selected. The procedure is described by Algorithm 3.

3. (Combination by ObjectiveCrossover, $\mathrm{C}_{\mathrm{O}}$ ) The third approach, is similar to what has been proposed in Laguna and Martí [23] for the knapsack problem. At most one variable can be active in each of the GUB sets. The choice of the variable in each GUB set is determined by a score $s$, calculated from the objective function value of the solutions as shown in Eq. 14:

$$
s=\frac{Z_{A}}{Z_{A}+Z_{B}} .
$$

For each GUB set, a random number $r$ is drawn. If $r \leq s$, then the partial solution of the first solution will be copied to the resulting solution. Otherwise, the partial solution of the second solution will be copied to the resulting solution. This procedure is described by Algorithm 4 .

4. (Combination by HybridCrossover, $\mathrm{C}_{\mathrm{H}}$ ) The fourth approach is similar to $\mathrm{C}_{\mathrm{O}}$, but it differs from $\mathrm{C}_{\mathrm{O}}$ in dealing with scenario 2. When a GUB set is active in both solutions, the variable with a bigger $\mathrm{B} / \mathrm{C}$ ratio will be selected with two-thirds of chance. This procedure is described by Algorithm 5 . 

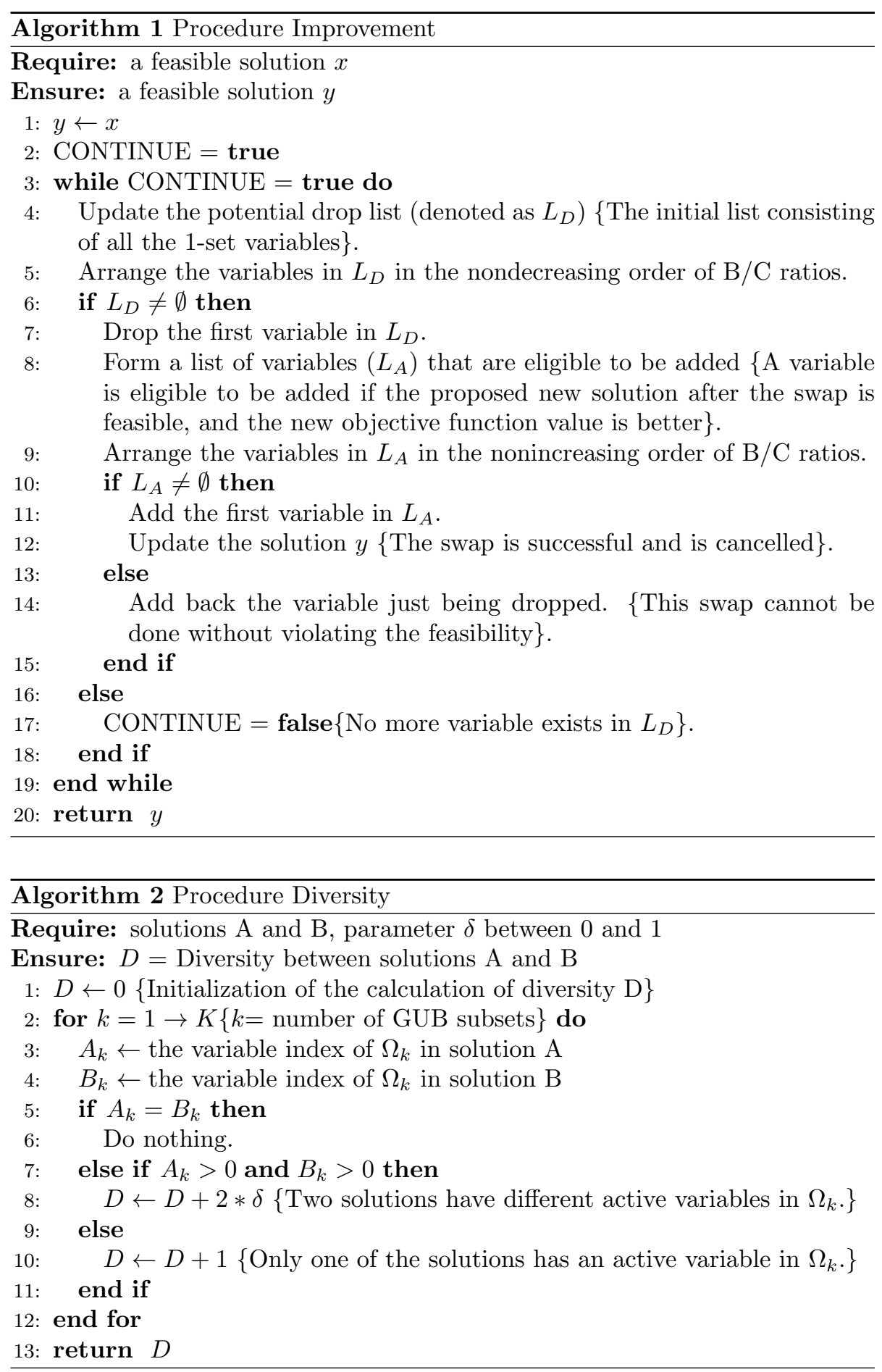


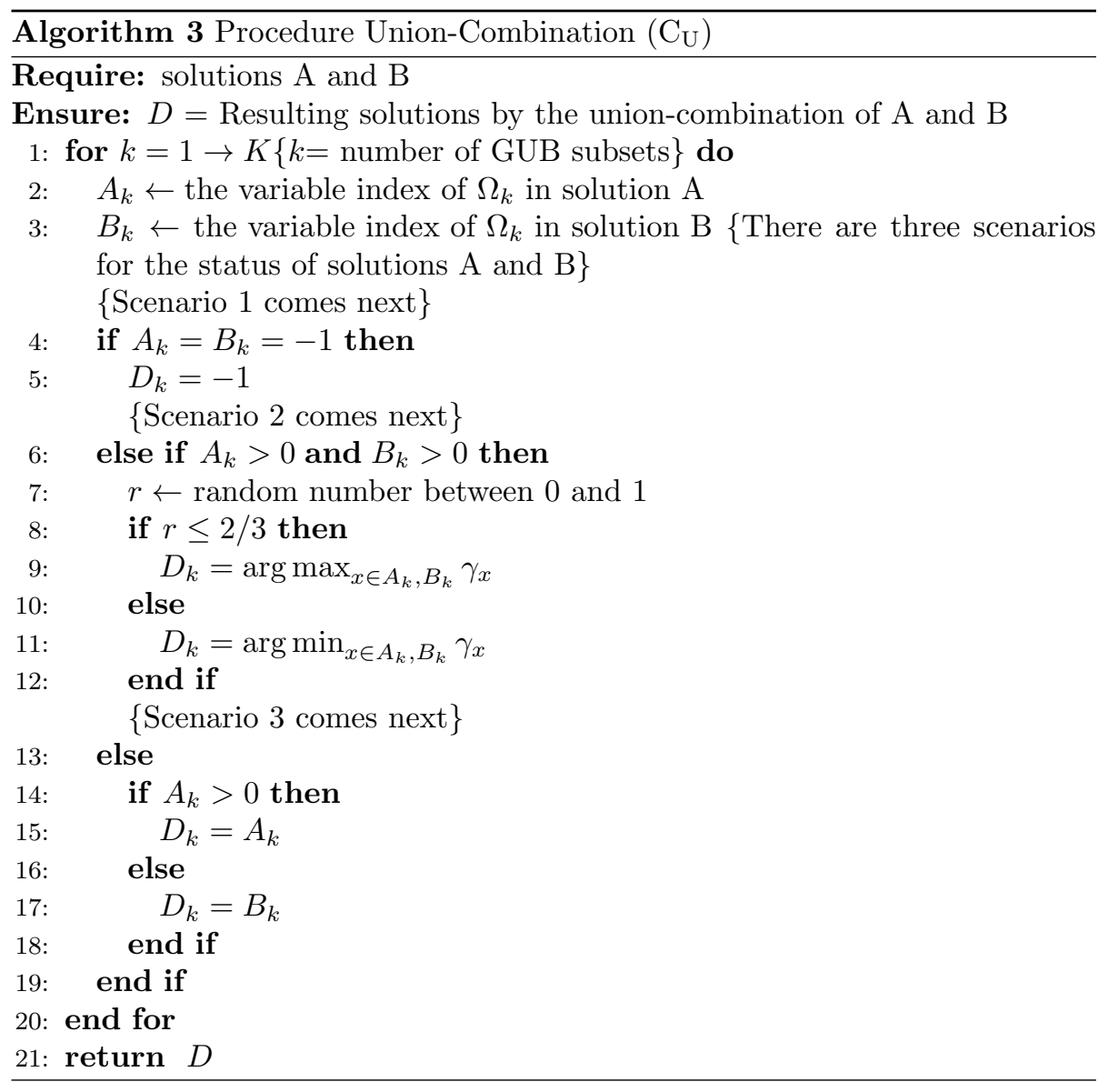

$\overline{\text { Algorithm } 4 \text { Procedure Objective-Crossover-Combination }\left(\mathrm{C}_{\mathrm{O}}\right)}$

\section{Require: solutions A and B}

Ensure: $D=$ Resulting solutions by the score-based-variable-combination of $\mathrm{A}$ and $\mathrm{B}$

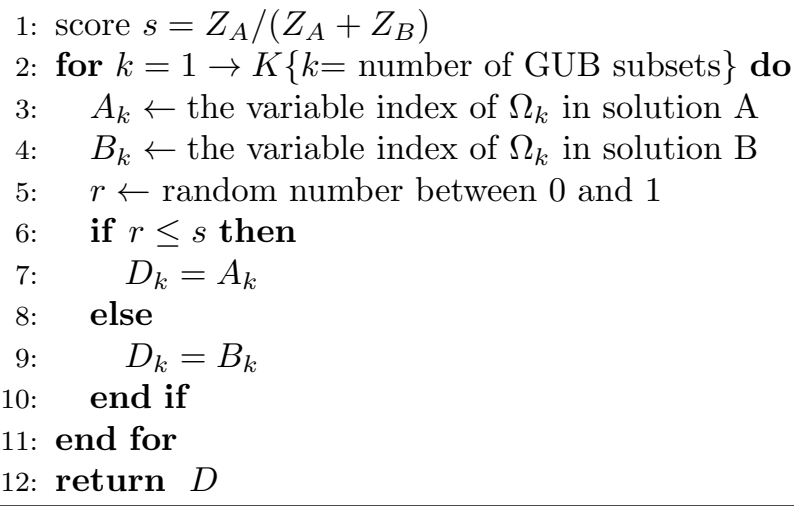




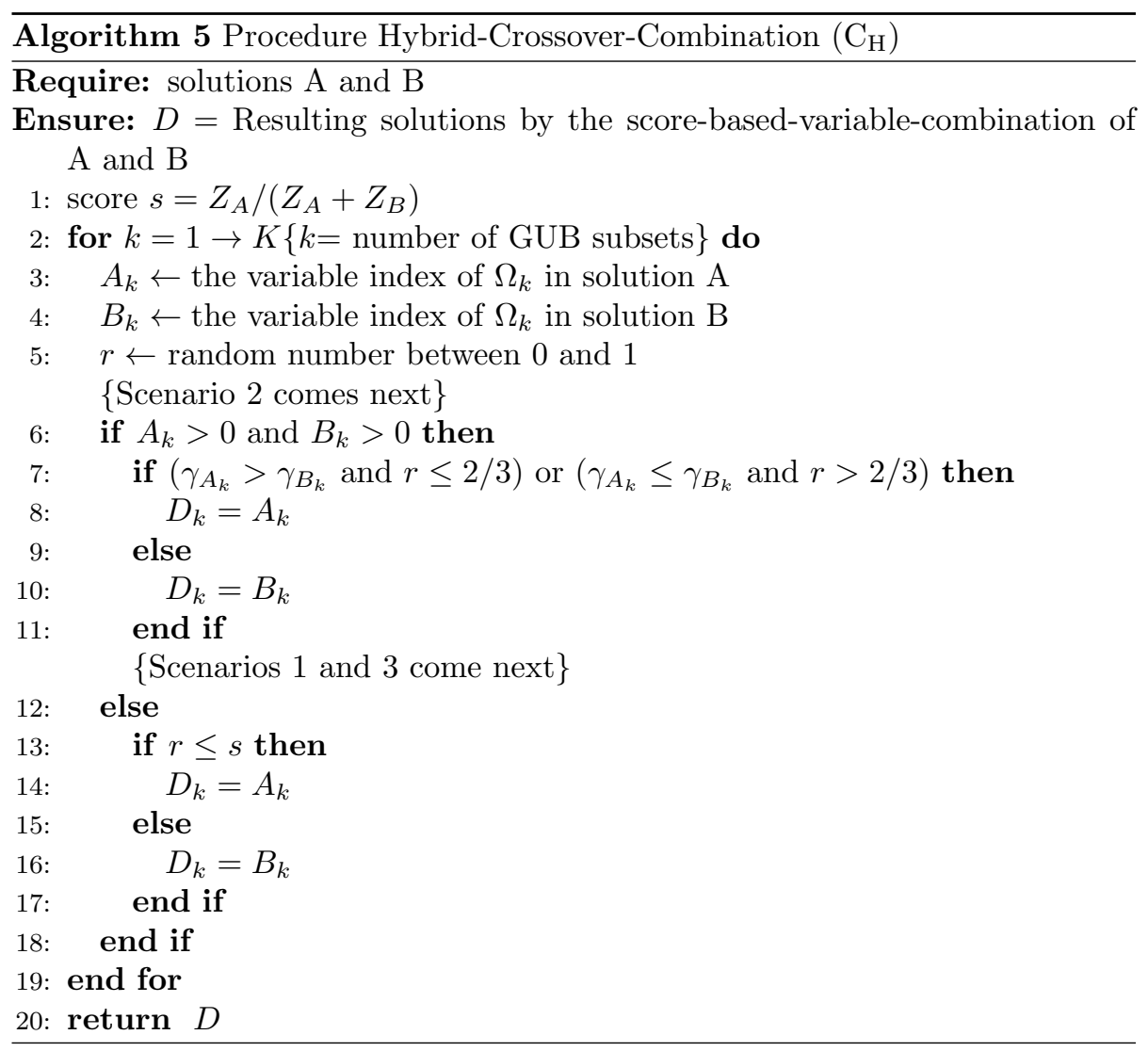


Notice that solutions obtained from $\mathrm{C}_{\mathrm{U}}, \mathrm{C}_{\mathrm{O}}$, or $\mathrm{C}_{\mathrm{H}}$ may violate some of the knapsack constraints. When this situation happens, the 1-set variable with the smallest B/C ratio will be dropped. This process will be repeated until the feasibility is restored. Last but not

\section{Computational and results}

The proposed method was coded in Visual C++ 2005 using a PC with Intel Core 2 Duo CPU E8500 3.16 GHz, 2.00 GB RAM and Windows XP operating system.

\subsection{Test problems}

The GUBMKP test set consists of 30 problem instances tested in $\mathrm{Li}$ [26]. The 30 test instances are equally partitioned into 3 categories of problems (referred as $\mathbf{S}, \mathbf{M}$, and $\mathbf{L}$ ) according to the number of knapsack constraints $(m)$ and the number of GUB sets $(K)$. The values of $(m, K)$ equal $(10,20),(20,40)$, and $(30,60)$ for $\mathbf{S}, \mathbf{M}$, and $\mathbf{L}$, respectively. There are about 4,000, 8,000, and 15,000 variables in the largest instance of $\mathbf{S}, \mathbf{M}$, and $\mathbf{L}$, respectively (they are referred to $\mathbf{S 1 0 0 ,} \mathbf{M 1 0 0}$, and $\mathbf{L 1 0 0 ,}$ respectively). For each category, problems were generated by taking a percentage of variables from the largest problem in the category. For example, S10 represents the smallest problem in the S-category, which takes approximately $10 \%$ of the variables from $\mathbf{S 1 0 0 .}$

\subsection{Parameter settings}

Typically, the initial pool size for the number of diversified solutions and the size of the reference set, according to the literature, are suggested no least, solutions obtained from combination will go through the improvement procedure as described in Section 3.2. With the five methods mentioned in Section 3, our scatter search can be represented using Figure 1.

more than 100 (see [16]) and 20 (see [30]), respectively. In our study, the initial pool and reference set were set to accommodate 100 and 20 solutions, respectively. The reference set was equally partitioned to subsets $R_{1}$ and $R_{2}$.

To test the heuristic, three strategy parameters were utilized described as follows:

1. Choosing diversity multiplier $(\delta)$ among 0, 0.25, 0.5, 0.75, and 1 .

2. Choosing the combination strategy in one of the following four methods: $\mathrm{C}_{\mathrm{I}}, \mathrm{C}_{\mathrm{U}}, \mathrm{C}_{\mathrm{O}}$ and $\mathrm{C}_{\mathrm{H}}$.

3. Choosing the $\alpha$ value for the GRASP. Comparison will be made among four discrete values including $0.6,0.7,0.8$, and 0.9 , and the uniform distribution among the previous four $\alpha$ values denoted as $\alpha_{u}$.

For each of the 30 problem instances, there are 100 (5 $\delta$ options $\times$ 4 combination strategies $\times 5 \alpha$ options) different combinations for these three strategy parameters. To simplify the computational work, five replications of 10-minute experiments were conducted for the largest problem instance (i.e., L100) under some combinations of these three parameters. The combination is based on the following three factors:

1. As for the $\delta$ value, it is truly hard to say why to ignore the dif- 


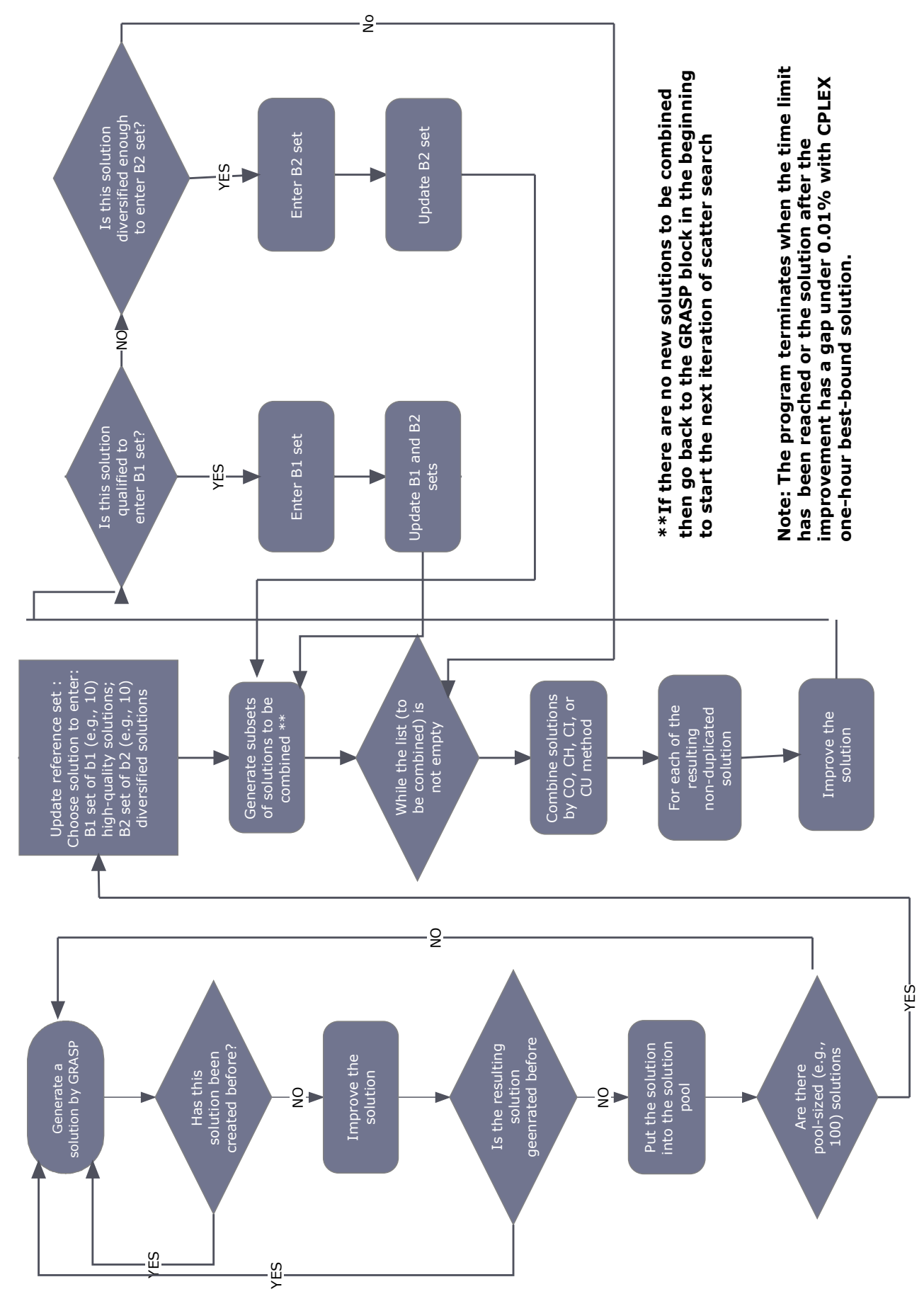

Figure 1: Scatter search flowchart for the GUBMKP 
Table 1: Average gap of $\mathbf{L 1 0 0}$ with different parameter setting

\begin{tabular}{crrr}
\hline \multirow{2}{*}{ Description of Experiments } & \multicolumn{3}{c}{ Average Gap (\%) } \\
& 1-min & 5-min & 10-min \\
\hline$\alpha=0.6$ & 3.43 & 2.99 & 2.87 \\
$\alpha=0.7$ & 3.06 & 2.80 & 2.74 \\
$\alpha=0.8$ & 3.17 & 2.75 & 2.53 \\
$\alpha=0.9$ & 2.99 & 2.60 & 2.30 \\
$\alpha_{\mathrm{u}}$ & 3.03 & 2.62 & 2.52 \\
\hline$\delta=0$ & 3.03 & 2.56 & 2.37 \\
$\delta=1$ & 3.20 & 2.94 & 2.79 \\
\hline $\mathrm{C}_{\mathrm{H}}$ & 2.94 & 2.61 & 2.46 \\
$\mathrm{C}_{\mathrm{O}}$ & 3.33 & 2.87 & 2.68 \\
$\mathrm{C}_{\mathrm{U}}$ & 3.08 & 2.79 & 2.60 \\
\hline
\end{tabular}

ference is more important than emphasizing the difference between solutions. Therefore, both boundary cases (i.e., 0 and 1 ) of $\delta$ are candidates.

2. In terms of the combination strategy, $\mathrm{C}_{\mathrm{I}}$ seems to be very restrictive since a variable cannot be 1 unless it is 1 in both solution. Therefore, we only consider the other three strategies (i.e., $\mathrm{C}_{\mathrm{U}}, \mathrm{C}_{\mathrm{O}}$, and $\mathrm{C}_{\mathrm{H}}$ ) for the base case choice.

These results help us to determine the base case setting by setting $\delta$ to 0 and $\mathrm{C}_{\mathrm{H}}$ as the combination strategy. However, for the $\alpha$ value, we observe the average gaps of $\alpha=0.9$ and $\alpha_{u}$ are very close when running 1 minute and 5 minutes. Setting $\alpha$ to 0.9 might be a little bit too risky in order to create good diversity for the solution pool, and eventually the reference set for some of the problems; therefore we chose to use $\alpha_{u}$ for the base case.

\subsection{Computational runs}

Based on the $\mathbf{L 1 0 0}$ preliminary results, $\delta$ is set to 0 , the combination
3. All five $\alpha$ choices are candidates.

\subsection{Preliminary results}

The results of these preliminary runs based on L100 are summarized in Table 1. The results shows (1) setting $\delta$ to 0 is better than setting it to 1, (2) choosing $\mathrm{C}_{\mathrm{H}}$ seems to be more promising than the other two combination strategies, and (3) setting $\alpha$ to 0.9 seems to perform best among the five options.

strategy is set to $\mathrm{C}_{\mathrm{H}}$, and $\alpha$ is set to $\alpha_{u}$ (the uniform distribution among 0.6, $0.7,0.8$, and 0.9$)$. By varying the strategy parameters of the base case one at a time, the following 12 cases including the base case were tested.

1. $\left(\alpha_{\mathrm{u}}, 0, \mathrm{C}_{\mathrm{H}}\right)$ : the base case

2. $\left(\alpha_{\mathrm{u}}, 0.25, \mathrm{C}_{\mathrm{H}}\right): \delta$ changes to 0.25

3. $\left(\alpha_{\mathrm{u}}, 0.5, \mathrm{C}_{\mathrm{H}}\right): \delta$ changes to 0.5

4. $\left(\alpha_{\mathrm{u}}, 0.75, \mathrm{C}_{\mathrm{H}}\right): \delta$ changes to 0.75

5. $\left(\alpha_{\mathrm{u}}, 1, \mathrm{C}_{\mathrm{H}}\right): \delta$ changes to 1

6. $\left(\alpha_{\mathrm{u}}, 0, \mathrm{C}_{\mathrm{I}}\right)$ : the combination strategy changes to $\mathrm{C}_{\mathrm{I}}$

7. $\left(\alpha_{\mathrm{u}}, 0, \mathrm{C}_{\mathrm{U}}\right)$ : the combination strategy changes to $\mathrm{C}_{\mathrm{U}}$ 
Table 2: 10-min. ave. gap (\%) of each class of problems with different $\delta$ values

\begin{tabular}{cccccc}
\hline Case No. & $\delta$ & S-instances & M-instances & L-instances & All-instances \\
\hline 1 & 0 & $0.01 \%$ & $1.55 \%$ & $2.23 \%$ & $1.26 \%$ \\
2 & 0.25 & $0.03 \%$ & $1.60 \%$ & $2.50 \%$ & $1.38 \%$ \\
3 & 0.5 & $0.03 \%$ & $1.71 \%$ & $2.61 \%$ & $1.45 \%$ \\
4 & 0.75 & $0.04 \%$ & $1.77 \%$ & $2.59 \%$ & $1.46 \%$ \\
5 & 1 & $0.02 \%$ & $1.78 \%$ & $2.63 \%$ & $1.48 \%$ \\
\hline
\end{tabular}

Table 3: 10-min. ave. gap (\%) of each class of problems with different combination strategies

\begin{tabular}{cccccc}
\hline Case No. & $\begin{array}{c}\text { Combination } \\
\text { Strategy }\end{array}$ & S-instances & M-instances & L-instances & All-instances \\
\hline 1 & $\mathrm{C}_{\mathrm{H}}$ & $0.01 \%$ & $1.55 \%$ & $2.23 \%$ & $1.26 \%$ \\
6 & $\mathrm{C}_{\mathrm{I}}$ & $0.17 \%$ & $1.96 \%$ & $2.56 \%$ & $1.56 \%$ \\
7 & $\mathrm{C}_{\mathrm{U}}$ & $0.05 \%$ & $1.76 \%$ & $2.02 \%$ & $1.28 \%$ \\
8 & $\mathrm{C}_{\mathrm{O}}$ & $0.02 \%$ & $1.61 \%$ & $2.29 \%$ & $1.31 \%$ \\
\hline
\end{tabular}

8. $\left(\alpha_{\mathrm{u}}, 0, \mathrm{C}_{\mathrm{O}}\right):$ the combination strategy changes to $\mathrm{C}_{\mathrm{O}}$

9. $\left(\alpha_{0.6}, 0, \mathrm{C}_{\mathrm{H}}\right): \alpha$ changes to 0.6

10. $\left(\alpha_{0.7}, 0, \mathrm{C}_{\mathrm{H}}\right): \alpha$ changes to 0.7

11. $\left(\alpha_{0.8}, 0, \mathrm{C}_{\mathrm{H}}\right): \alpha$ changes to 0.8

12. $\left(\alpha_{0.9}, 0, \mathrm{C}_{\mathrm{H}}\right): \alpha$ changes to 0.9

To evaluate the quality of the solution, the best objective function value from the SS heuristic $\left(Z_{\mathrm{SS}}\right)$ was compared to the CPLEX (version 11.2.1) one-hour upper bound value $\left(Z_{\mathrm{BB}}\right)$. Eq. 15 shows how to calculate the percentage gap.

$$
\mathrm{GAP}=\frac{Z_{\mathrm{BB}}-Z_{\mathrm{SS}}}{Z_{\mathrm{BB}}} * 100 \%
$$

Each of these 12 cases were tested for 10 minutes for three times due to the random nature of GRASP, and the average gap for each problem instance was calculated. We further calculated the average gap of each category $(\mathbf{S}$, $\mathbf{M}$, and $\mathbf{L}$ ) of problem instances. Other things being equal, the results (the average gap of 30 instances) of these tests showed

1. $0 \succcurlyeq 0.25 \succcurlyeq 0.50 \succcurlyeq 0.75 \succcurlyeq 1$ when varying the $\delta$ value (Table 2 );

2. $\mathrm{C}_{\mathrm{H}} \succcurlyeq \mathrm{C}_{\mathrm{U}} \succcurlyeq \mathrm{C}_{\mathrm{O}} \succcurlyeq \mathrm{C}_{\mathrm{I}}$ for the combination strategy (Table 3);

3. $0.9 \succcurlyeq 0.8 \succcurlyeq \alpha_{\mathrm{u}} \succcurlyeq 0.7 \succcurlyeq 0.6$ when varying the $\alpha$ value (Table 4 ).

Table 4: 10-min. ave. gap (\%) of each class of problems with different $\alpha$ value

\begin{tabular}{cccccc}
\hline Case No. & $\alpha$-value & S-instances & M-instances & L-instances & All-instances \\
\hline 9 & 0.6 & $0.01 \%$ & $1.76 \%$ & $2.60 \%$ & $1.46 \%$ \\
10 & 0.7 & $0.01 \%$ & $1.67 \%$ & $2.65 \%$ & $1.44 \%$ \\
11 & 0.8 & $0.01 \%$ & $1.47 \%$ & $2.11 \%$ & $1.20 \%$ \\
12 & 0.9 & $0.05 \%$ & $1.46 \%$ & $1.90 \%$ & $1.14 \%$ \\
1 & $\alpha_{\mathrm{u}}$ & $0.01 \%$ & $1.55 \%$ & $2.23 \%$ & $1.26 \%$ \\
\hline
\end{tabular}


The first observation on the value of $\delta$ reveals that it is more important to count the difference between two solutions due to different states of a GUB set. If the active GUB sets of the two base solutions are the same, then the difference within each GUB set may be ignored. The second observation on the combination strategy indicates that the resulting solution obtained from taking the intersection approach might be too conservative. The hybrid approach and the union approach seem to show relative strength for different class of problems (e.g., hybrid is best for $\mathbf{M}$-instances while union is best for L-instances).

The third observation bases on the $\alpha$ value shows the more greedy approach in the GRASP part, the better the resulting solution is. However, when the scale of problem becomes relatively small (e.g., S-instances), the quality of the most greedy approach becomes inferior to all other options. That is because the choices for GRASP become more limited when the problem size shrinks. Therefore, it is probably safer to use a relatively random approach when facing small problems, and use a more greedy approach when

\section{Conclusions}

In this paper, we have developed a scatter search heuristic for the GUBMKP that follows the fivecomponent template proposed by Glover [11]. First of all, this heuristic uses GRASP to generate a diversified pool of solutions. Experimental results showed drawing the $\alpha$ value randomly among some fixed values leads to more balanced performance over instances. The solutions are improved using swap dealing with larger problems. Nevertheless, if the $\alpha$ value becomes too large, regardless the problem size, it might negatively affect the diversity of the reference pool as well as the overall solution quality of scatter search.

Based on the 10-minute allinstances average gap among these 12 cases, we select cases 1,11 , and 12 to represent our scatter search heuristic to compare with several methods in the literature including critical event tabu search (CETS) with local search [26], tight oscillation (TO) [24], and adaptive memory projection (AMP) [27]. The comparison in Table 5 shows the AMP approach has attained the best results overall. Nevertheless, when comparing the S-instances (small instances), all three SS cases perform equally or better than the AMP approach in 5- and 10-minute run time limits. In addition, when considering the performance in 1-minute run time, case 12 of SS performs equally to the H15 case of AMP over different size of instances. Also note that case 12 of SS is superior to the H15 case of AMP in the $\mathbf{L}$-instances which indicates that SS can converge to high quality solution at early stage for large instances.

moves based on $\mathrm{B} / \mathrm{C}$ ratios. Secondly, in order to choose solutions to enter the diversity reference subset, a diversity algorithm was proposed to measure the differences between two solutions. As solution can be different in a few ways, the algorithm parameter $\delta$ can be varied to weight all sorts of differences. Finally, four combination mechanisms have been developed. The hybrid-crossover combination $\left(\mathrm{C}_{\mathrm{H}}\right)$ is most promising among these four alternatives. The results obtained on 
Table 5: Comparison of average gaps among various heuristics for the GUBMKP

\begin{tabular}{cccrr} 
Method & S-instances & M-instances & L-instances & All instances \\
\hline & Run time limit (1-min) & & \\
\hline CETS with local search & 2.66 & 8.84 & 12.92 & 8.14 \\
TO & 0.46 & 2.39 & 3.27 & 2.04 \\
AMP (H15) & 0.11 & 1.86 & 2.95 & 1.64 \\
SS (case 1) & 0.12 & 2.30 & 2.89 & 1.77 \\
SS (case 11) & 0.04 & 2.15 & 2.93 & 1.70 \\
SS (case 12) & 0.15 & 2.15 & 2.64 & 1.64 \\
\hline & Run time limit (5-min) & & \\
\hline CETS with local search & 2.04 & 7.75 & 11.78 & 7.19 \\
TO & 0.38 & 2.00 & 2.77 & 1.72 \\
AMP (H15) & 0.08 & 1.25 & 1.10 & 0.81 \\
SS (case 1) & 0.03 & 1.73 & 2.41 & 1.39 \\
SS (case 11) & 0.02 & 1.67 & 2.35 & 1.35 \\
SS (case 12) & 0.08 & 1.65 & 2.19 & 1.31 \\
\hline & Run time limit (10- min) & & \\
\hline CETS with local search & 2.01 & 7.13 & 11.35 & 6.83 \\
TO & 0.38 & 1.94 & 2.59 & 1.64 \\
AMP (H15) & 0.07 & 1.20 & 0.83 & 0.70 \\
SS (case 1) & 0.01 & 1.55 & 2.23 & 1.26 \\
SS (case 11) & 0.01 & 1.47 & 2.11 & 1.20 \\
SS (case 12) & 0.05 & 1.46 & 1.90 & 1.14 \\
\hline
\end{tabular}

a classic set of GUBMKP instances show our scatter search heuristic outperforms the tight oscillation method and the critical event tabu search with local search, and also performs competitively to the adaptive memory projection method that has the best computational results for this class of problems.

A future extension to this study is to have a reference set with three subsets. The diversity measure can be differentiated by evaluating the difference from the GUB-set or variable perspectives by setting $\delta$ to 0 and 1 in Algorithm 2, respectively. Alternatively, it is worth trying to let the third subset include infeasible solutions that are close to the feasibility boundary.

\section{Acknowledgements}

The authors would like to thank both reviewers for their valuable comments. We are also grateful to Dr. ChiLeung Chu for providing suggestions on the revision. Funding for the first author was provided by the National Science Council of Taiwan (R.O.C.) under grant number 95-2221-E-259022 .

\section{About the Contributors}

Vincent C. Li is an Associate Professor in the Department of Business Administration at National Chiayi University in Taiwan. Dr. Li received his MS and PhD degrees in Industrial Engineering from Texas A\&M University. His research interests include 
metaheuristics, operations research, logistics, and supply chain management.

Yun-Chia Liang received his first MS (Mechanical Engineering, 1996) from Carnegie Mellon University, a second MS (Industrial Engineering, 1999) from University of Pittsburgh, and a $\mathrm{PhD}$ in Industrial and Systems Engineering from Auburn University (2001). His research interests are in application of metaheuristics to optimization problems. Dr. Liang has authored over 100 publications in books, refereed proceedings and journals including articles in IEEE Transactions on Reliability, Computers and Operations Research, Computers and Industrial Engineering, European Journal of Operational Research, and Journal of Heuristics. Dr. Liang is currently an Associate Professor in the Department of Industrial Engineering and Management at Yuan Ze University, Taiwan. He is a member of IEEE, CIIE, IAENG, and ORSTW.

Yu-Yen Sun is currently an Engineer in the UI Framework Department at HTC in Taiwan. He received his bachelor degree in Computer Science and Information Engineering from $\mathrm{Na}$ tional Dong Hwa University in 2009. Mr. Sun is interested in algorithm design and implementation.

You-Shan Chen is a chief of the manufacturing department in Yeong Shyh Cheng Alu Company Limited in Taiwan. He received his bachelor degree in Industrial Engineering from Chung Yuan Christian University (Taiwan), and MS degree in Global Operations Strategy and Logistics Management from National Dong Hwa University (Taiwan). His research interests include metaheuristics, scheduling and supply chain management.

(Received October 2011; revised July 2012; accepted October 2012)

\section{References}

[1] Beausoleil, R., Baldoquin, G., Montejo, R., 2008. Multi-start and path relinking methods to deal with multiobjective knapsack problems. Annals of Operations Research 157 (1), 105-133.

[2] Chardaire, P., McKeown, G., Maki, J., 2001. Application of GRASP to the multiconstraint knapsack problem. In: Boers, E. (Ed.), Applications of Evolutionary Computing. Vol. 2037 of Lecture Notes in Computer Science. Springer Berlin / Heidelberg, Germany, pp. 30-39.

[3] Crévits, I., Hanafi, S., Mansi, R., Wilbaut, C., 2012. Iterative semi-continuous relaxation heuristics for the multiple-choice multidimensional knapsack problem. Computers \& Operations Research 39 (1), 32-41, special Issue on Knapsack Problems and Applications.

[4] Feo, T. A., Resende, M. G., 1995. Greedy randomized adaptive search procedures. Journal of Global Optimization 6, 109-133.

[5] Florios, K., Mavrotas, G., Diakoulaki, D., 2010. Solving multiobjective, multiconstraint knapsack problems using mathematical programming and evolutionary algorithms. European Journal of Operational Research 203 (1), 14 21. 
[6] Fréville, A., 2004. The multidimensional 0-1 knapsack problem: An overview. European Journal of Operational Research 155 (1), 121.

[7] Gholipour-Kanani, Y., TavakkoliMoghaddam, R., Khorrami, A., 2011. Solving a multi-criteria group scheduling problem for a cellular manufacturing system by scatter search. Journal of the Chinese Institute of Industrial Engineers 28 (3), 192-205.

[8] Gilmore, P., Gomory, R., 1966. The theory and computation of knapsack functions. Operations Research 14 (6), 1045-1075.

[9] Glover, F., 1977. Heuristics for integer programming using surrogate constraints. Decision Sciences 8 (1), 156-166.

[10] Glover, F., 1998. Surrogate Constraints: Tutorial Notes. Graduate School of Business, University of Colorado, Boulder, Colorado, USA.

[11] Glover, F., 1998. A template for scatter search and path relinking. In: Hao, J.-K., Lutton, E., Ronald, E., Schoenauer, M., Snyers, D. (Eds.), Artificial Evolution, Lecture Notes in Computer Science 1363. Springer, London, UK, pp. 13-54.

[12] Glover, F., 2000. Multi-start and strategic oscillation methodsprinciples to exploit adaptive memory. In: Laguna, M., Velarde, J. L. G. (Eds.), Computing Tools for Modeling, Optimization and Simulation: Interfaces in Computer Science and Operations Re- search. Kluwer Academic Publishers, Boston, USA, pp. 1-24.

[13] Glover, F., 2005. Adaptive memory projection methods for integer programming. In: Rego, C., Alidaee, B. (Eds.), Metaheuristic Optimization via Memory and Evolution: Tabu Search and Scatter Search. Kluwer Academic Publishers, Boston, USA, Ch. 19, pp. 425-440.

[14] Glover, F., Kochenberger, G., 1996. Critical event tabu search for multidimensional knapsack problems. In: Osman, I. H., Kelly, J. P. (Eds.), Metaheuristics: Theory and Applications. Kluwer Academic Publishers, Boston, USA, pp. 407-427.

[15] Glover, F., Laguna, M., Martí, R., 2000. Fundamentals of scatter search and path relinking. Control and Cybernetics 29 (3), 653-684.

[16] Glover, F., Laguna, M., Martí, R., 2003. Scatter search. In: Ghosh, A., Tsutsui, S. (Eds.), Advances in Evolutionary Computation: Theory And Applications. Springer, New York, pp. 519-537.

[17] Gomes da Silva, C., Clímaco, J., Figueira, J., 2004. A scatter search method for the bicriteria multi-dimensional $\{0,1\}$ knapsack problem using surrogate relaxation. Journal of Mathematical Modelling and Algorithms 3 (3), 183-208.

[18] Gomes da Silva, C., Clímaco, J., Figueira, J., 2006. A scatter search method for bi-criteria $\{0$, $1\}$-knapsack problems. European Journal of Operational Research 169 (2), 373-391. 
[19] Hanafi, S., Wilbaut, C., 2008. Scatter search for the 0-1 multidimensional knapsack problem. Journal of Mathematical Modelling and Algorithms 7 (2), 143159 .

[20] Hiremath, C., 2008. New heuristic and metaheuristic approaches applied to the multiple-choice multidimensional knapsack problem. Ph.D. thesis, Wright State University, Dayton, Ohio, USA.

[21] Hiremath, C. S., Hill, R. R., 2007. New greedy heuristics for the multiple-choice multi-dimensional knapsack problem. International Journal of Operational Research 2 (4), 495-512.

[22] Hvattum, L. M., Lokketangen, A., 2007. Experiments using scatter search for the multidemand multidimensional knapsack problem. In: Doerner, K. F., Gendreau, M., Greistorfer, P., Gutjahr, W., Hartl, R. F., Reimann, M., Sharda, R., Vo, S. (Eds.), Metaheuristics. Vol. 39 of Operations Research/Computer Science Interfaces Series. Springer, New York, pp. 3-24.

[23] Laguna, M., Martí, R., 2002. Scatter Search: Methodology and Implementations in C. Kluwer Academic Publishers, Boston, USA.

[24] Li, V. C., 2005. Tight oscillations tabu search for multidimensional knapsack problems with generalized upper bound constraints. Computers \& Operations Research 32 (11), 2843-2852.
[25] Li, V. C., Curry, G., Boyd, E., 2004. Towards the real time solution of strike force asset allocation problems. Computers \& Operations Research 31 (2), 273-291.

[26] Li, V. C., Curry, G. L., 2005. Solving multidimensional knapsack problems with generalized upper bound constraints using critical event tabu search. Computers \& Operations Research 32 (4), 825-848.

[27] Li, V. C., Liang, Y. C., Chang, H. F., 2012. Solving the multidimensional knapsack problems with generalized upper bound constraints by the adaptive memory projection method. Computers \& Operations Research 39 (9), 2111-2121.

[28] Lust, T., Teghem, J., 2012. The multiobjective multidimensional knapsack problem: a survey and a new approach. International Transactions in Operational Research 19 (4), 495-520.

[29] Martello, S., Toth, P., 1990. Knapsack Problems. Wiley, New York, USA.

[30] Martí, R., Laguna, M., Glover, F., 2006. Principles of scatter search. European Journal of Operational Research 169 (2), 359-372.

[31] Meier, H., Christofides, N., Salkin, G., 2001. Capital budgeting under uncertainty-an integrated approach using contingent claims analysis and integer programming. Operations Research 49 (2), 196-206.

[32] Moser, M., Jokanovic, D., Shiratori, N., 1997. An algorithm 
for the multidimensional multiplechoice knapsack problem. IEICE Transactions on Fundamentals of Electronics Communications and Computer Sciences E80-A (3), 582-589.

[33] Peterson, C., 1967. Computational experience with variants of the Balas algorithm applied to the selection of research and development projects. Management Science 13 (9), 736-750.

[34] Prais, M., Ribeiro, C. C., 2000. Reactive GRASP: An application to a matrix decomposition problem in tdma traffic assignment. INFORMS Journal on Computing 12 (3), 164-176.

[35] Resende, M., Ribeiro, C., 2003. Greedy randomized adaptive search procedures. In: Glover, F., Kochenberger, G. (Eds.), Handbook of Metaheuristics. Vol. 57 of International Series in Operations Research \& Management Science. Springer, New York, pp. 219-249.
[36] Shih, W., 1979. A branch and bound method for the multiconstraint zero-one knapsack problem. Journal of Operations Research Society 30 (4), 369-378.

[37] Vasquez, M., Hao, J.-K., 2001. A logic-constrained knapsack formulation and a tabu algorithm for the daily photograph scheduling of an earth observation satellite. Computational Optimization and Applications 20 (2), 137-157.

[38] Wilbaut, C., Hanafi, S., Salhi, S., 2008. A survey of effective heuristics and their application to a variety of knapsack problems. IMA Journal of Management Mathematics 19 (3), 227-244.

[39] Wilhelm, W., Gokce, E., 2010. Branch-and-price decomposition to design a surveillance system for port and waterway security. IEEE Transactions on Automation Science and Engineering 7 (2), 316325 . 